\title{
Multi-parametric surface plasmon resonance platform for studying liposome-serum interactions and protein corona formation
}

\section{Kari, Otto K.}

2017

Kari , O K, Rojalin , T , Salmaso , S, Barattin , M , Jarva , H, Meri , S, Yliperttula , M , Viitala , T \& Urtti , A 2017 , ' Multi-parametric surface plasmon resonance platform for studying liposome-serum interactions and protein corona formation ', Drug Delivery and Translational Research , vol. 7 , no. 2 , pp. 1-13 . https://doi.org/10.1007/s13346-016-0320-0

http://hdl.handle.net/10138/309640

https://doi.org/10.1007/s13346-016-0320-0

unspecified

acceptedVersion

Downloaded from Helda, University of Helsinki institutional repository.

This is an electronic reprint of the original article.

This reprint may differ from the original in pagination and typographic detail.

Please cite the original version. 


\title{
Multi-Parametric Surface Plasmon Resonance Platform for Studying Liposome-Serum Interactions and Protein Corona Formation
}

Otto K. Kari1;, Tatu Rojalin1», Stefano Salmaso2, Michela Barattin2, Hanna Jarva3, Seppo Meriz, Marjo Yliperttula1, Tapani Viitala $1^{*}$, Arto Urtti1,4

${ }_{1}$ Centre for Drug Research and Division of Pharmaceutical Biosciences, Faculty of Pharmacy, University of Helsinki, Viikinkaari 5, P.O. Box 56, FI-00014, Finland

2 Department of Pharmaceutical and Pharmacological Sciences, University of Padova, Via F. Marzolo 5, 35131 Padova, Italy

3 Department of Bacteriology and Immunology, Immunobiology Research Program, Faculty of Medicine, and

HUSLAB, Division of Clinical Microbiology, Haartmaninkatu 3, P.O. Box 21, FI-00014, Finland

4School of Pharmacy, University of Eastern Finland, Yliopistonranta 1, FI-70211 Kuopio, Finland

$\$$ O.K.K and T.R. contributed equally to this work

*Corresponding author: Dr. Tapani Viitala (e-mail: tapani.viitala@ helsinki.fi, phone +358 (0) 2941 59626)

\section{Keywords}

Multi-parametric surface plasmon resonance (MP-SPR); protein corona; soft corona; liposome; complement system; opsonin

\section{Acknowledgements}

We are grateful to M.Sc. Tatu Lajunen, B.Sc. Riikka Nurmi and M.Sc. Antti Louna for the help in preparing and characterizing the control liposome formulations, and to Leena Pietilä and Dr. Mari Palviainen for the help with serum collection and pooling. Liposomes coated with a lipidated oligo-guanidyl derivative were kindly supplied by Professor Stefano Salmaso, University of Padova. Financial support by the Academy of Finland (grants: \#137053, \#263861, \#263567), Tekes - the Finnish Funding Agency for Innovation EV-Extra-Tox project and the Professor Pool - Orion Research Foundation are gratefully acknowledged.

\begin{abstract}
When nanocarriers are administered into blood circulation, a complex biomolecular layer known as the 'protein corona' associates with their surface. Although the drivers of corona formation are not known, it is widely accepted that this layer mediates biological interactions of the nanocarrier with its surroundings. Label-free optical methods can be used to study protein corona formation without interfering with its dynamics. We demonstrate the proof-of-concept for a multiparametric surface plasmon resonance (MP-SPR) technique in monitoring the formation of a protein corona on surfaceimmobilized liposomes subjected to flowing $100 \%$ human serum. We observed the formation of formulation dependent 'hard' and 'soft' coronas with distinct refractive indices, layer thicknesses and surface mass densities. MP-SPR was also employed to determine the affinity $\left(\mathrm{K}_{D}\right)$ of a complement system molecule $(\mathrm{C} 3 \mathrm{~b})$ with cationic liposomes with and without polyethylene glycol. Tendency to create a thick corona correlated with a higher affinity of opsonin C3b for the surface. The label-free platform provides a fast and robust preclinical tool for tuning nanocarrier surface architecture and composition to control protein corona formation.
\end{abstract}

\section{Introduction}

Nanomedicine has advanced several research fields (e.g. cancer and gene therapy, medical imaging) by enabling the targeted delivery of therapeutic and diagnostic molecules while protecting the sensitive cargo [1,2]. Hydrophilic molecules, such as polyethylene glycol (PEG), are used to shield nanocarriers from unspecific protein binding that may change biodistribution, decrease cellular uptake, and lead to premature nanocarrier clearance by the mononuclear phagocyte system (MPS) [3]. In fact, PEG can increase the average circulation time of liposomal drugs from minutes to up to days [3]. However, it is widely accepted that the steric hindrance provided by PEG and other hydrophilic coatings does not abrogate unspecific protein binding, and these coatings can act as immune activators [4-7]. Since severe 
hypersensitivity reactions to nanocarriers have been reported in clinical use, improved preclinical methods to assess the safety of nanocarriers are needed [2,4,5,8-10]. In particular, label-free methods to predict biological interactions of nanocarriers are warranted, as labels may lead to biased results [2,11]. The safety of nanocarriers can be improved by decreasing the immunogenicity of nanocarriers, or by increasing their cellular targeting, both of which are affected by the proteins adsorbed on the nanocarrier surface.

After nanocarrier introduction into blood circulation, their surface is rapidly covered with a complex and dynamic layer of biomolecules such as proteins, lipids and other plasma components [3,12-14]. This layer is often referred to as the "protein corona" and it is further divided into a stable "hard corona" and a diffuse secondary layer of proteins, the "soft corona" [3,12-14]. Following the pioneering work of Cedervall et al.[14] to characterize the kinetics of protein corona formation on nanoparticle surfaces, protein-nanocarrier interactions have received increasing attention $[15,16]$. At present, the influence of the protein corona on the fate of the nanocarriers in the body has been clearly demonstrated $[3,11,13,17]$. Proteomic characterization of the protein corona has helped to identify nanocarrier-specific protein fingerprints - not a simple task considering the highly dynamic nature of the protein corona [15,17-20]. More recently, the focus has shifted towards exploiting protein corona formation for extracellular and intracellular targeting, understanding its evolution, and its role in mediating physiological responses to nanocarriers $[8,15,17,20]$. However, there is still a need to increase our understanding of the protein corona phenomenon and its dynamics due to its biological significance.

The prevailing hypothesis on the dynamics of corona formation assumes the formation of a highly dynamic but slowlydeveloping hard corona with a limited number of proteins [11,14,18]. This paradigm was challenged by Tenzer et al. [17], who reported the formation of a highly complex and particle-specific protein corona on all nanoparticles tested in 30seconds. The rapid formation and composition of the protein coronas could not be explained merely by the physicochemical properties of the nanoparticles, and at longer incubation times, the protein composition changed only quantitatively but not qualitatively [17]. According to the authors, the rapid formation of a protein fingerprint is sensible considering the highly dynamic nature of physiological systems, which have evolved to "react instantly" to preserve homeostasis. The effect of dynamic incubation on protein corona formation was recently demonstrated with PEGmodified liposomes when static incubation was compared to a flow conditions mimicking blood flow dynamics [12]. Over 200 corona proteins were identified, with 108 proteins common to both incubation conditions, whereas 10 and 99 proteins were observed only after incubation in static or dynamic conditions, respectively [12]. For increased in vivo relevance, the protein corona formation should therefore be tested in flow conditions.

The studies by Palchetti et al.[12] and Tenzer et al.[17] indicate the presence of complement system proteins in the corona. This is not surprising considering the nature of these proteins as the probes of the immune system, and the non-allergic hypersensitivity reactions that have been reported following the administration of PEGylated liposomes (e.g. Doxilø) that has been linked to complement system activation [5,9]. For instance, covalent deposition of C3b on the surface of nanocarriers lead to an increased MPS clearance rate and a tendency to activate an innate immune response [3,21,22]. $\mathrm{C} 3 \mathrm{~b}$ is an activated opsonin formed from the central complement protein $\mathrm{C} 3$, at which all three activation pathways converge for amplification [21]. Complement proteins can carry out their functions by binding both to the native nanocarrier surface or through attachment to surface-bound proteins such as albumin [23]. Therefore, for reduced immunogenicity, nanocarrier surfaces should not favor the binding of $\mathrm{C} 3 \mathrm{~b}$ or other complement components [5,9,21].

Most analytical techniques are not suitable to study the soft corona on nanocarriers $[12,16,17,20]$. Biophysical analytical approaches to monitor the soft corona could provide important information, and there is a growing interest in Raman spectroscopy, quartz crystal microbalance (QCM) and surface plasmon resonance (SPR) to study the evolution of corona formation in real-time $[16,20]$. The SPR phenomenon has been widely used for biosensing applications, including the interaction kinetics of individual proteins and complement system components on biomaterial surfaces [24]. Previous SPR studies on the interactions of nanocarriers with blood proteins have employed diluted plasma $[13,23,25]$. The surface of biosensors can be functionalized with specific chemistries of interest, proteins, or a hydrogel and lipid matrix that can be used to capture nanocarriers on the sensor surface - an approach we utilize in this work among others [14,25-27].

SPR permits the sensitive label-free monitoring of molecular interactions [28,29]. It is based on propagating waves of surface plasmons produced on a metal surface upon excitation with light. The conditions for exciting the surface plasmons are dependent on the refractive index of the medium in contact with the metal surface, and the molecular mass changes 
on the metal surface. In essence, the binding of molecules such as proteins to the metal surface can be detected as a change in the SPR peak angular position, OSPR. Figure 1 illustrates a set of idealized full SPR angular spectra; the solid red and blue traces representing measured spectra, and the black and orange dotted lines showing calculated counterparts for the red and blue spectra, respectively. The main components of spectra are marked as (1) the angular position of total internal reflection (TIR) event, and (2) the angular position of SPR peak minimum, referred to as $\theta$ sPR in this manuscript. As the analytes are deposited onto the sensor surface, a shift towards larger angular values takes place in the $\theta$ SPR (Figure 1, (2) ). However, the angular position of TIR may also undergo a change towards larger values if the refractive properties of running buffer and injected analyte differ significantly (Figure 1, (1)). This bulk effect, in turn, can be compensated for in the $\theta$ SPR and thus the remaining part of the angular change mainly represents the binding event at the surface. In general, the full SPR angular spectra contain all the physical information to solve for the layer thicknesses and refractive indices; the shape and quantities associated with the SPR curve are utilized. A more profound mathematical description of solving for the layer thickness and refractive index is given in Materials and Methods section. Lastly, it is worthwhile pointing out, that the full SPR angular spectra are used to solve for deposited layer thicknesses and refractive indices whereas the sensorgrams are utilized for determining the interaction kinetics parameters. Importantly, in the sensorgrams the $\theta$ SPR (displayed in Figure 1 as red and blue dots) is followed in real time which allows for calculating the kinetic parameters if the injected analyte concentrations are known.

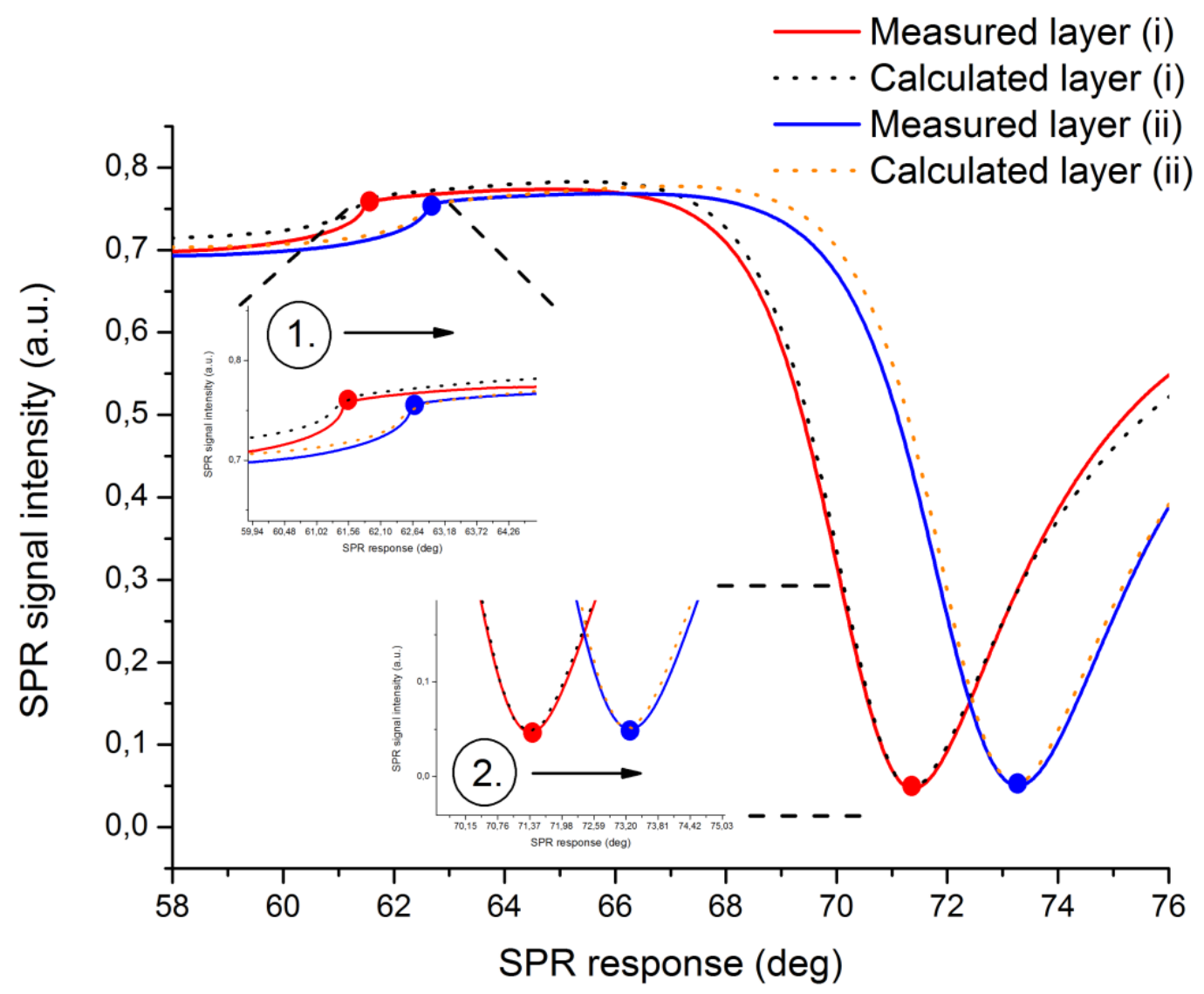

Figure 1. An idealized example of two measured full SPR angular spectra (red and blue solid curves) and the fitted spectra (black and orange dotted curves, respectively). The shape and quantities associated with the SPR curve are used to fit the measured data and consequently, solve for surface-deposited layer thicknesses $(d)$ and refractive indices $(n)$. The calculation is carried out in a step-wise numerical iteration routine whereby the parameters $(d$ and $n)$ for each layer are calculated utilizing the information provided by two independent excitation wavelengths $(670 \mathrm{~nm}$ and $785 \mathrm{~nm})$. For clarity, instead of displaying four measured curves, spectra recorded from one wavelength are depicted in the figure. The shift in angular position of total internal reflection (TIR) is used to compensate for bulk effect caused by the differences between running buffer and injected sample; e.g. serum injection into the flow system consisting of running buffer induces a significant change in the TIR angle (1). In order to estimate the signal for the binding event to the surface, the TIR shift contribution can be compensated by a subtraction from the overall signal. In general, binding events are detected as a shift in $\theta$ SPR (2)). 
Multi-parametric surface plasmon resonance (MP-SPR) refers to an adaptation of the SPR technology that can be used to optically characterize biomolecular layers in more detail and with thicknesses of up to several micrometers [28] (Figure 1). Traditionally, the incident angle of minimum reflectance has been monitored over a narrow angular area near the $\theta$ sPR. With the MP-SPR technique, the full SPR angular spectrum is recorded by scanning over a wide angular range ( 40-78 degrees) and multiple parameters can be acquired simultaneously; for example the angular position of the TIR angle, the angular position of $\theta$ SPR, the half-width of the main SPR peak and the change in the main SPR peak intensity at $\theta$ sPR can be monitored in real-time. The combination of two or more wavelengths and a scanning angle feature makes it possible to record the entire SPR angular spectrum as a function of time. Unlike with other SPR instruments that employ only one wavelength, the MP-SPR adaptation allows for determination of layer thicknesses, refractive indices and surface mass densities in addition to traditional kinetic association and dissociation events [28,30,31]. These features provide new opportunities for a more detailed surface characterization of protein coronas, which we have explored in this work. In this paper, we describe the proof-of-concept for a MP-SPR method to monitor the formation of protein coronas on nanocarriers in real-time at the interface of flowing undiluted serum. We also demonstrate the use of MP-SPR to determine the affinity of a complement system probe molecule $\mathrm{C} 3 \mathrm{~b}$ interaction with the sensor-immobilized liposome surface.

\section{Materials and Methods}

Serum preparation. Blood drawn from seven healthy and fasted donors using Vacutainer glass tubes without clot activator (BD, Franklin Lakes, NJ, USA) was left to clot for $30 \mathrm{~min}$ at $22^{\circ} \mathrm{C}$, followed by centrifugation at $2500 \times \mathrm{g}$ for $5 \mathrm{~min}$ and $3500 \times \mathrm{g}$ for $5 \mathrm{~min}$. The serum fractions were collected manually and pooled together with serum from other donors in aseptic conditions, and stored in aliquots at $-80^{\circ} \mathrm{C}$. Before SPR measurements, aliquots were thawed rapidly in a $37^{\circ} \mathrm{C}$ water bath until $90 \%$ thawed. Heat homogeneity was checked with a FLIR TG165 imaging IR thermometer (FLIR Systems, Inc., OR, USA) to avoid bubble formation inside the SPR flow channel due to temperature changes.

Liposome samples. The film re-hydration method followed by extrusion through a polycarbonate membrane was used to prepare liposome samples [32]. Control liposomes were replicas of the Doxil@ (Johnson and Johnson, NJ, USA; marketed in Europe under the name Caelyx $\odot$ by Janssen-Cilag International NV, Beerse, Belgium) formulation with and without PEG (referred to as DOX+PEG and DOX, respectively) but without doxorubicin[32]. The molar ratio of fully hydrogenated soy phosphatidylcholine (HSPC), cholesterol and N-(carbonyl-methoxypolyethylene glycol 2000)-1,2distearoyl-sn-glycero-3-phosphoethanolamine sodium salt (DSPE-PEG2000) or 1,2-distearoyl-sn-glycero-3phosphoethanolamine (DSPE) was 55:40:5, respectively[32]. After preparation the DOX and DOX+PEG liposome stock suspensions were stored at $4^{\circ} \mathrm{C}$ until use. For SPR measurements, the DOX and DOX+PEG liposome samples were adjusted to a $3 \mathrm{mM}$ total lipid concentration.

Positively charged liposomes with oligo-guanidyl lipid derivative (OGD) with a $0.2 \mathrm{mg} / \mathrm{ml}$ total lipid concentration in HEPES $10 \mathrm{mM}$ and $\mathrm{NaCl} 150 \mathrm{mM}$ (pH 7.4) buffer solution were freeze dried after preparation and stored at $-20^{\circ} \mathrm{C}$ until use[33]. For SPR measurements, the OGD liposomes samples were quickly thawed and vortexed. The OGD liposomes adsorbed on the sensor surface were then coated in situ according to a charge-to-charge association process with a polyanionic-PEG block-copolymer (PEG) with a concentration of $0.1085 \mathrm{mg} / \mathrm{ml}$ in HEPES $10 \mathrm{mM}$ and $\mathrm{NaCl} 150 \mathrm{mM}$ (pH 7.4) buffer solution (OGD+PEG).

Endotoxin detection. The presence of endotoxins was tested using Limulus Amoebocyte Lysate (LAL) assays: Pierce LAL chromogenic endotoxin quantitation kit (Thermo Fisher Scientific, Waltham, MA, USA) and Pyrogent Plus Gel Clot LAL at $0.06 \mathrm{EU} / \mathrm{ml}$ sensitivity (Lonza, Basel, Switzerland). Assays were performed according to manufacturers' instructions using endotoxin-free consumables. Test samples were incubated using a Thermomixer $\mathrm{C}$ heating block (Eppendorf AG, Hamburg, Germany). Absorbance (405 nm) was measured using a Spectrostar Nano plate reader (BMG Labtech, Ortenberg, Germany) and calculated using the accompanying MARS software. Inhibition and enhancement controls and transmissivity measurements of the liposome solution at assay wavelength were performed to rule out liposome interference with the LAL assays.

Physicochemical characterization of liposome samples. Hydrodynamic diameter measurements were performed at 25 ${ }^{\circ} \mathrm{C}$ with a Zetasizer APS DLS automated plate sampler (Malvern Instruments, Malvern, United Kingdom). Results are reported as size distributions (average hydrodynamic diameter \pm S.D.) derived from relative signal intensities and in the 
form of a polydispersity index (PDI). Surface charge of the liposomes was determined by measuring the $\zeta$-potential in DTS 1070 folded capillary cells (Malvern Instruments) by using a Zetasizer Nano ZS instrument (Malvern Instruments).

MP-SPR measurements. Measurements were performed with a SPR Navitм 200 (BioNavis Ltd., Ylöjärvi, Finland) instrument. The setup was equipped with two incident laser wavelengths, $670 \mathrm{~nm}$ and $785 \mathrm{~nm}$, two independent flow channels, inlet tubing and outlet (waste) tubing. Both of the flow channels were measured in parallel with $670 \mathrm{~nm}$ and $785 \mathrm{~nm}$ incident light. The measurement temperature was kept constant at $20{ }^{\circ} \mathrm{C}$, and the flow rates used for liposome immobilization and for serum interactions were $50 \mu \mathrm{L} / \mathrm{min}$ and $100 \mu \mathrm{L} / \mathrm{min}$, respectively. Liposomes were captured and immobilized on SPR sensors consisting of a thin $6 \mathrm{kD}$ carboxymethyl dextran hydrogel layer functionalized with dodecyl lipid anchors [26,34]. The SPR sensors were used repeatedly after rejuvenation with an injection series of Hellmanex II 2\% (Hellma Analytics, Müllheim, Germany) or CHAPS $20 \mathrm{mM}$ (Sigma-Aldrich), ethanol 80\% (Altia Oyj, Rajamäki, Finland) and deionized water from a Milli-Q water purification system (Merck KGaA, Darmstadt, Germany). In between measurements, the sensors were stored immersed in CHAPS at $4^{\circ} \mathrm{C}$. During the SPR measurements, the functionalized gold sensor slides were first subjected to the running buffer for respective liposome for approximately 5-10 minutes until a stable baseline was achieved. In the second phase, the liposomes were injected into both flow channels for 10 minutes, and lastly, sensor surfaces containing the immobilized liposome species were subjected to $100 \%$ serum. The PEGylation step for the OGD liposomes was performed in situ with a polyanionic-PEG block-copolymer after liposome immobilization to create a surface with OGD+PEG, whereas DOX+PEG liposomes contained PEG prior to immobilization.

MP-SPR data analysis. The shape and quantities associated with the SPR curve are utilized for the layer thickness and refractive index calculations. The SPR spectrum can be described with Fresnel's equations as the reflectivity of a multilayered system for p-polarized light [28,30]. In essence, the layers are assumed linear, homogenous, and isotropic. The calculation is based on a 2 X 2 scattering matrix that is derived from Fresnel's complex-amplitude reflection and transmission coefficients. The matrix represents the summarized optical properties of the system; the properties are expressed as a product of the interface and layer matrices of the entire structure. Furthermore, the intermediate zones between different layers are introduced into Fresnel's equations as new layers. Thus, the thickness of the intermediate layer describes the average roughness of the surface whereas its complex refractive index corresponds to the light losses via scattering and plasmon decoupling into radiative modes (essentially the imaginary part of the complex refractive index). The benefit of this approach is that only Fresnel's equations can be used without the need to apply e.g. scattering theories. In practice, the modeling and solving for the matrix formalism is performed by mathematical fitting tools or dedicated software. To solve for the layer thicknesses and refractive indices of the thin biomolecular layers, a twowavelength method combined with angular scanning over a wide range of incident light angles is particularly efficient [35-37]. The measured full angular SPR spectrum and matrix formalism are utilized to model the layers. Even though the overall mathematics behind the calculations is relatively complicated, the solving process can be simplified to a level that the modeling still produces accurate results for most of the situations that deal with common materials and biological molecules. Additionally, the sources for error are feasible to track due to the known limitations of these evaluations. It is worth emphasizing that the multi-wavelength approach for surface characterization has been successfully implemented in previous studies $[26,31,38,39]$.

The general solution for a multilayered, optically active system that is connected with measurable and controllable quantities, can be derived using the aforementioned transfer matrix formalism of 2 X 2 matrices. In the complex refractive index $\hat{n}=n+i k$ the real part $\mathrm{n}$ corresponds to the refraction of light, and the complex coefficient $\mathrm{k}$ to the extinction or absorption of light by the material. Furthermore, the relationship $\hat{n}=\sqrt{\hat{\varepsilon}}$ connects the refractive index and dielectric constant. Finally, in practical experiments, the surface plasmon wave vector $\left(\mathrm{k}_{\mathrm{sp}}\right)$ is considered to contain all the information and constants that affect the measured SPR spectra, and lead to differences in SPR spectra measured at multiple wavelengths. Therefore, the continuum solution for surface plasmon wave vector $\left(\mathrm{ksp}_{\mathrm{sp}}\right)$ in one set of conditions can be deducted to describe the mathematical relationship between ( $\mathrm{ksp}$ ), the material layer thickness $\mathrm{d}$, and the refractive index n:

$$
\mathrm{k}_{s p} \propto n * d
$$

However, the definition above describes only one continuum solution for the ( $\mathrm{ksp}$ ). The unique solution for the apparently interconnected thickness $\mathrm{d}$ and refractive index $\mathrm{n}$ of the sample layer build in the given experimental conditions can be 
found from the intersection of two continuum solutions. Therefore a measurement in two different media or multiple wavelengths is needed [28,31]. In the mathematical treatment of the two-wavelength method, the refractive index $n$ has also a wavelength dependency, $\mathrm{dn} / \mathrm{d} \lambda$. The unique solution for layer thickness $\mathrm{d}$ and refractive index $\mathrm{n}$ can be found by solving for the equations:

$$
\begin{aligned}
& k_{s p 1}=n_{\lambda 1} * d \\
& k_{s p 2}=n_{\lambda 2} * d
\end{aligned}
$$

where $k_{s p 1}$ denotes the surface plasmon vector for wavelength $1, n_{\lambda 1}$ the refractive index for wavelength $1, k_{s p 2}$ the surface plasmon vector for wavelength 2 , and $n_{\lambda 2}$ the refractive index for wavelength 2 , and:

and:

$$
n_{\lambda 2}=n_{\lambda 1}+\frac{d n}{d \lambda} *\left(\lambda_{2}-\lambda_{1}\right)
$$

$$
\begin{gathered}
k_{s p 1}=n_{\lambda 1} * d \\
k_{s p 2}=\left(n_{\lambda 1}+\frac{d n}{d \lambda} *\left(\lambda_{2}-\lambda_{1}\right)\right) * d
\end{gathered}
$$

The statements above hold true for sample layers that do not absorb light at the wavelengths used in the SPR spectrum measurement. In practice, it means that the imaginary component $\mathrm{k}$ for the refractive index $=0$, which is the case for the majority of organic sample layers. However, if the layer absorbs light, $k \neq 0$, and there is a unique solution for the sample layer in the $k_{s p}=(n+i k) * d$ space. One can utilize laser wavelengths $670 \mathrm{~nm}$ and $785 \mathrm{~nm}$ that are aligned to irradiate simultaneously the same spot in each sample channel. The thicknesses of modeled layers can be linked as common for both wavelengths in the modeling step, i.e. the layer thickness is expected to be the same for both wavelengths since the SPR angular spectra are measured from the same spot. The complex refractive index can be input as an independent variable for background (e.g. pure metal layer) modeling, or as a linearly dependent variable between the two used wavelengths for reasons described above.

The SPR Navi LayerSolver v. 1.2.1 (BioNavis Ltd., Ylöjärvi, Finland) software was used for the layer thickness $(d)$ and refractive index $(n)$ modeling and calculations. In essence, the modeling and calculation of layer thickness and refractive index were carried out using the two-wavelength method. The LayerSolver allows for multiple SPR spectra processing, and a step-wise approach for calculations where the biophysical properties of the molecular layers are determined by numerical iteration one layer at a time. The software was used to simultaneously solve the thickness and refractive index based on full SPR angular spectra measured with two different wavelength lasers (670 nm and $785 \mathrm{~nm})$ at the same time point $[28,30]$. The numerical iteration is performed by the embedded algorithms in LayerSolver software. The equations described in section "MP-SPR data analysis" constitute the physical cornerstone for the calculation routines in LayerSolver. As input variables, the following layer thickness and refractive index values were used: for OGD, $d=45$ $70 \mathrm{~nm}$ and $\mathrm{n}=1.357-1.355$; for DOX, $\mathrm{d}=80-120 \mathrm{~nm}$ and $\mathrm{n}=1.357-1.352$; for OGD and DOX soft corona, $\mathrm{d}=20-70 \mathrm{~nm}$ and $n=1.37-1.38$; for OGD and DOX hard corona $d=5-10 \mathrm{~nm}$ and $\mathrm{n}=1.38-1.39$. In the optical modeling performed in this work, the thickness values were defined as global variables for the two wavelengths used and the refractive indices were defined as independent variables for the iteration cycles.

Interaction kinetics were modeled with TraceDrawer v. 1.6 (Ridgeview Instruments AB, Vänge, Sweden) software. The calculation of interaction parameters is based on well-established kinetic models [40]. Herein, a one binding site model was used for determining the affinity of the human native complement component C3b (diluted in Dulbecco's phosphate buffered saline, obtained from Merck KGaA, Darmstadt, Germany) interacting with immobilized OGD or OGD+PEG liposomes. According to the supplier, the $\mathrm{C} 3 \mathrm{~b}$ protein used should display at least $60 \%$ of its physiological activity. A concentration series $0.00833,0.0833$, and $0.833 \mathrm{~mol}$ (determined with a MW of $180 \mathrm{kDa}$ from $0.0015,0.015$, and 0.15 $\mathrm{mg} / \mathrm{ml}$ ) fitting with negligible dissociation phases was performed for the measured C3b sensorgrams in order to calculate the kinetic parameters for C3b. In general, molecular mass that binds onto the SPR sensor surface has a well-defined correlation with the observed SPR angular spectrum and the SPR resonance angle shift in particular. Hence, the surface mass densities of $\mathrm{C} 3 \mathrm{~b}$ can be calculated by using the de Feijter equation that is derived from the definition of refractive 
index increment of adsorbing biomolecules, with some limitations [41,42]. For the used SPR Naviтм 200 instrument and $670 \mathrm{~nm}$ laser wavelength, the actual surface mass density conversion coefficient can be defined using a value of $d n / d c$ $=0.182 \mathrm{~cm}_{3} / \mathrm{g}$. In this study, to convert the observed SPR signal responses to surface mass densities $(\Gamma)$, we used the conversion factor $\Delta 1 \mathrm{mdeg}=\Delta(\Gamma) \sim 0.6 \mathrm{ng} / \mathrm{cm} 2$ for the lower limit of deposited surface mass density. To estimate the error in the calculation, we also defined the higher limit using the conversion factor $\Delta 1 \mathrm{mdeg}=\Delta(\Gamma) \sim 1.0 \mathrm{ng} / \mathrm{cm} 2$.

\section{Results and Discussion}

Liposome immobilization and serum interaction with the immobilized liposomes. Figure 1 illustrates the measurement scheme for serum interaction studies with the liposomes. Undiluted serum was used instead of anticoagulated plasma since it contains a functioning complement system. Roman numerals are used consistently to refer to the modeled physical events. Figures 2 and 4 (A and B), and Figures 3 and 5 (A and B) display the MP-SPR sensorgrams and full SPR angular spectra, respectively, with: (I) the liposome layer after immobilization onto the sensor surface, (II) serum interaction with liposomes and ensuing soft corona formation, and (III) the hard corona after restoration of buffer flow. In this work, the term soft corona is used to refer to the combination of a denser corona layer accompanied by a more diffuse layer of weakly interacting proteins. The averages of the modeled and calculated layer thicknesses $(d)$ and refractive index $(n)$ values of three independently repeated measurements are found in Table 1 for OGD and OGD+PEG liposomes and in Table 2 for DOX and DOX+PEG liposomes. In order to emphasize the highly dynamic behavior of the modeled systems, to take into account the possible sensitivity of differential equations to initial conditions provided as inputs in LayerSolver, and to estimate the uncertainties in experimental procedures and calculations, we chose to define a range for the calculated $(d)$ and $(n)$ values. Thus, instead of representing one set of $(d)$ and $(n)$ values, our intention was to determine the dlow and nlow as well as dhigh and nhigh, between which the calculated values could potentially vary. The dlow, nlow, dhigh, and nhigh values were calculated by using a consistent set of initial values throughout the layer modeling. Table S1 presents the results of physicochemical characterization (size, PDI and $\zeta$-potential) and endotoxin determination. The results for each individual experiment are found in Table S2 for OGD and OGD+PEG liposomes and Table S3 for DOX and DOX+PEG liposomes. In order to show that the observed events are not dependent on the flow channel used, the immobilized liposome species were interchanged between the flow channels from experiment to experiment.
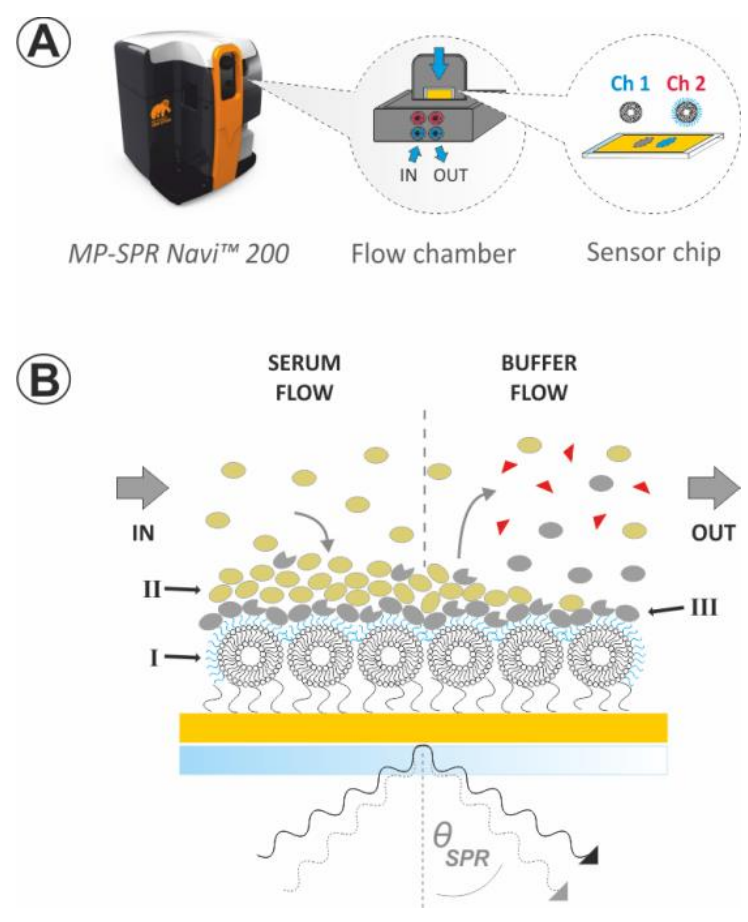
Fig. 2 Schematic representation of the experimental setup using a MP-SPR Navi ${ }^{\mathrm{TM}} 200$ instrument (instrument image courtesy of BioNavis Ltd., Ylöjärvi, Finland). A: Liposomes were captured from inflow and immobilized onto functionalized sensors. Measurements were run in parallel with the same serum source to compare the effect of hydrophilic coating molecules on protein corona formation. B: Simplified representation of the sensor surface to demonstrate the distinct layers of interest in the modelling step. Roman numerals indicate (I) the OGD liposome layer after immobilization onto the sensor and in situ functionalization with the polyanionic-PEG block-copolymer (blue), (II) serum interaction with liposomes and following soft corona formation, and (III) the hard corona after restoration of buffer flow.

OGD and OGD+PEG liposome interaction with 100\% serum. Figure $3 \mathrm{~A}$ shows the real-time sensorgrams from one measurement demonstrating OGD liposome immobilization onto the SPR sensor in both channels, followed by the PEGylation step in channel 2 ( $\mathrm{CH} 2)$ at $\mathrm{t} 30 \mathrm{~min}$ to form OGD+PEG. In channel 1 (CH1) with plain OGD liposomes, the signal remains at a constant level during running buffer injection. Figure 3B and Table 1 present the serum interaction results.
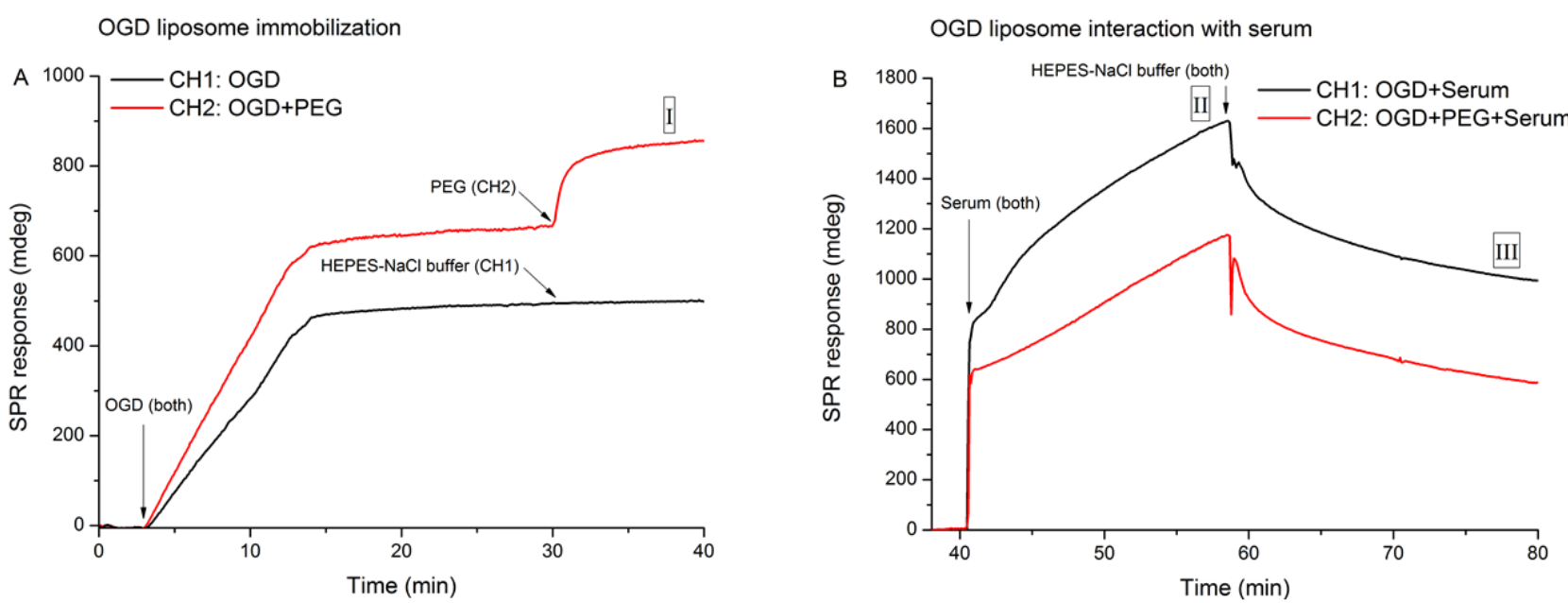

Fig. 3 MP-SPR sensorgrams for immobilization of OGD liposomes and their interaction with serum. A: Liposome immobilization to both channels with PEG modification in situ at $\mathrm{t} \sim 30 \mathrm{~min}$ for $\mathrm{CH} 2$. Thickness $(d)$ and refractive index $(n)$ of liposome layers have been modeled and calculated at time point I. B: Serum interaction (from 40-60 min) with the immobilized OGD (CH1) or OGD+PEG (CH2) liposomes at a flow speed of $100 \mu \mathrm{L} / \mathrm{min}$. Thickness $(d)$ and refractive index $(n)$ were modeled and calculated at II for soft corona, and at III for hard corona after restoration of buffer flow.

Figure 4 (A and B) present the full SPR spectra from real-time experiments for OGD and OGD+PEG liposomes at time points when the upper and lower boundaries for layer thicknesses and refractive indices were determined. First, the functionalized gold sensor surface with a $6 \mathrm{kDa}$ carboxymethyl dextran hydrogel matrix with dodecyl lipid anchors was measured and modeled (black line), followed by the (I) immobilized liposome layer, (II) soft corona and (III) hard corona. For clarity, the measured metal layer and OGD or OGD+PEG liposome layers are displayed without the fitted curves, whereas the soft and hard corona layers are depicted with both measured and fitted curves. Based on the step-wise numerical iteration procedure in LayerSolver, where the previous layer acts as a background to solve for the thickness and refractive index of the consequent layer, the modeling yields fairly good alignment between the calculated and measured SPR spectra throughout the procedure. Typically, the SPR angular peak minimum tends to shift towards larger angles as molecules (mass) adsorbing onto the surface and consequently change the refractive index in close vicinity to the surface. Thus, the surface plasmon coupling condition undergoes a change that can be observed as a shift in the peak minimum angular position [27,43,44]. The biomolecular layer build-up is clearly seen in Figure 4 (insets in A and B) where the SPR peak minimum shifts from the value observed for the metal (black traces) to the right when liposomes are associating with the surface (red traces) and both the total internal reflection (TIR) angle and the SPR peak minimum shifts further to the right when a soft corona forms (blue solid trace and the corresponding fitted purple dashed line). Interestingly, when the hard corona layer is revealed after restoration of buffer flow, the peak minimum value shifts back to the left (green solid traces and the navy blue dashed fitted lines) - which is an expected phenomenon when serum with a much higher refractive index is replaced by a buffer solution with lower refractive index (affects mainly TIR angular 
position) and when components of the looser soft corona layer dissociate, leaving a denser layer on the surface of immobilized liposomes.

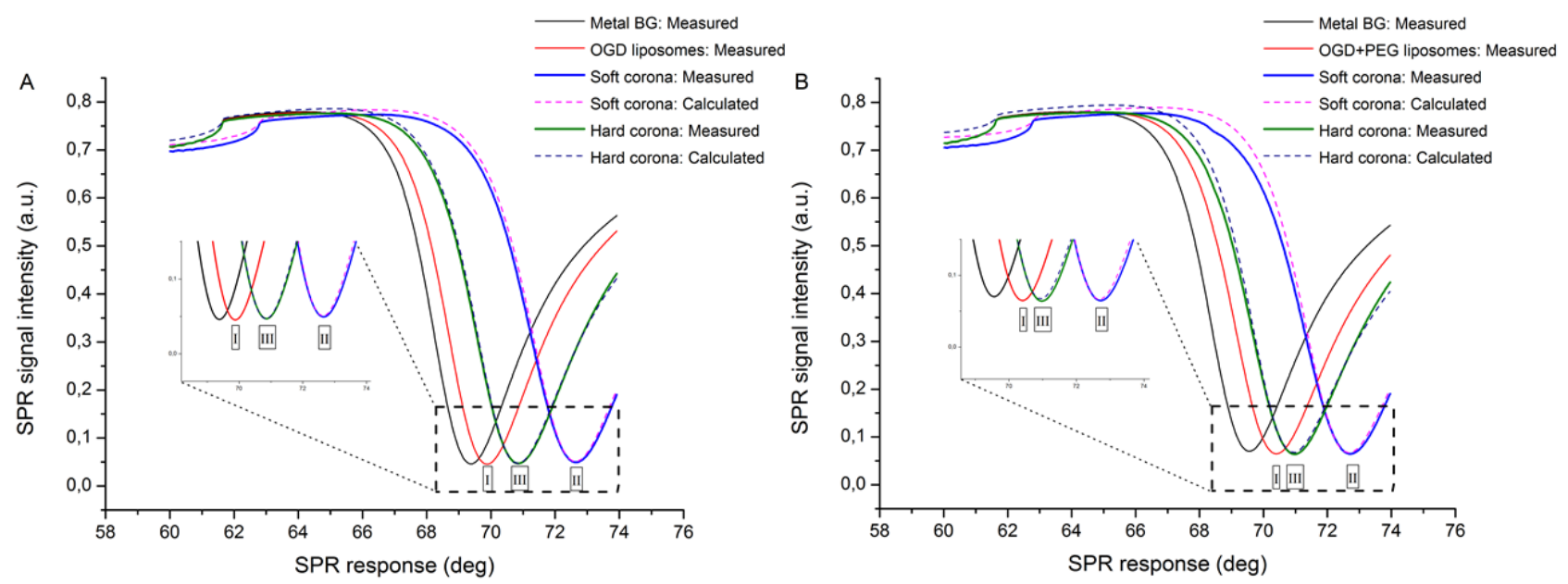

Fig. 4 Layer modeling for OGD and OGD+PEG liposomes using the measured full SPR angular spectra. A: SPR peak minimum shifts for the measured and modeled phases from the metal sensor surface (black line) to plain OGD (I) liposome immobilization, (II) soft corona, and (III) hard corona. B: Corresponding events for OGD+PEG.

Table 1. OGD and OGD+PEG liposomes and serum interaction results. The calculated values are low and high limits for layer thickness $(d)$ with corresponding refractive index $(n)$ values as averages ( \pm standard deviation) of three independent measurements for: (I) immobilized OGD liposomes with and without PEG modification; (II) soft protein corona formation following serum interaction; and (III) the residual hard corona after buffer flow restoration when a stable baseline is observed.

\begin{tabular}{|c|c|c|c|c|c|c|c|}
\hline & & \multicolumn{2}{|c|}{ (I) Liposome } & \multicolumn{2}{|c|}{ (II) Soft corona } & \multicolumn{2}{|c|}{ (III) Hard corona } \\
\hline \multirow{3}{*}{ 잉 } & & Low & High & Low & High & Low & High \\
\hline & $d$ & $40.4 \pm 9.3$ & $55.5 \pm 16.0$ & $34.2 \pm 2.1$ & $106.0 \pm 17.4$ & $9.6 \pm 1.0$ & $16.4 \pm 0.9$ \\
\hline & $n$ & $\begin{array}{l}1.35302 \\
\pm 0.0021\end{array}$ & $\begin{array}{c}1.34996 \\
\pm 0.00151\end{array}$ & $\begin{array}{c}1.39701 \\
\pm 0.0080\end{array}$ & $\begin{array}{c}1.37742 \\
\pm 0.0016\end{array}$ & $\begin{array}{c}1.44627 \\
\pm 0.0072\end{array}$ & $\begin{array}{c}1.41121 \\
\pm 0.0035\end{array}$ \\
\hline & & Low & High & Low & High & Low & High \\
\hline 产 & $d$ & $44.4 \pm 4.2$ & $60.9 \pm 8.1$ & $22.2 \pm 14.5$ & $63.2 \pm 57.2$ & $4.6 \pm 2.7$ & $8.6 \pm 6.4$ \\
\hline ఫે & $n$ & $\begin{array}{c}1.35679 \\
\pm 0.00292\end{array}$ & $\begin{array}{c}1.35263 \\
\pm 0.00215\end{array}$ & $\begin{array}{c}1.37931 \\
\pm 0.0178\end{array}$ & $\begin{array}{r}1.38013 \\
\pm 0.0071\end{array}$ & $\begin{array}{c}1.39524 \\
\pm 0.0393\end{array}$ & $\begin{array}{c}1.37531 \\
\pm 0.0227\end{array}$ \\
\hline
\end{tabular}

As shown in Table 1, the immobilized OGD liposomes form on average a slightly thinner layer on the sensor surface (40.4-55.5 nm) compared to the PEGylated OGD layer (44.4-60.9 nm), with basically the same refractive index for both systems. The slightly larger thickness for the ODG+PEG layer can be attributed to the additional PEG layer deposited on the adsorbed OGD liposome layer, whereas the additional PEG layer will not significantly affect the refractive index due to the high water content in the PEG layer. Although MP-SPR signal response levels exceeding 500 mdeg suggest that saturation of the sensor was achieved by both OGD and OGD+PEG, it is noteworthy that the SPR curve produced by MP-SPR is an average of the entire sensor surface; factors such as the non-homogeneous placement of liposomes, gaps in the layers, or the formation of multilayers can also influence the layer thicknesses and refractive indices observed [45]. Morphological changes in the shape of the liposome due to the interactions with the functionalized sensor surface and the shear stress from buffer flow are also expected, and they account for the differences to hydrodynamic diameters measured in bulk (Table S1).

Following serum injection, a thicker soft corona layer (34.2-106.0 nm) forms on the plain OGD liposome layer compared to the OGD+PEG liposome layer $(22.2-63.2 \mathrm{~nm})$. The plain OGD liposome layer presents surface charges that favor the association of passing serum components. Moving beyond the presumption that steric PEG protection decreases protein 
adsorption to liposome surfaces, we hypothesize that this observation can be explained by higher opsonisation and possible surface-induced activation of the complement system by the OGD liposome bearing oligo-guanidyl moieties and traces of endotoxin (Table S1) [12,21,24]. Complement activation leads to the recruitment of other complement components and formation of enzyme convertases that amplify the activation process by catalyzing opsonin production and their surface deposition in high quantities, thereby producing more molecular mass on the surface [21,24]. The tendency to activate the complement system is influenced by the amount and orientation of PEG molecules on the surface of liposomes [5,46,47]. However, the standard deviation of soft corona layer thickness in the case of OGD+PEG is relatively large ( \pm 57.2 at upper boundary) and a more definitive conclusion warrants further studies. Despite the difference in soft corona layer thicknesses, refractive indices in the same range for OGD and OGD+PEG suggest a similar consistency, presumably related to the more diffuse and rapidly exchanging nature of the soft corona layer at the vicinity of the surface. Finally, when a buffer rinse is performed after serum injection, a significantly thicker and denser hard corona layer remains on the surface of the plain OGD liposomes compared to OGD+PEG, which may report of complement activation and deposition of covalently bound complement end products onto the surface. To our knowledge, this is the first time when MP-SPR is used to optically characterize properties of protein corona layers. These results provide clear support for the existence of a distinct, more diffuse soft corona layer on top of the hard corona [13,17,20].

DOX and DOX+PEG liposome interaction with 100\% serum. Figure 5 and 6 show the real-time sensorgrams and the full angular SPR spectra, respectively, for DOX and DOX+PEG liposomes. The layer thicknesses and refractive indices are shown in Table 2. In Figure 4A, DOX liposomes without PEG were immobilized to CH1 and DOX+PEG to CH2. Since DOX+PEG liposomes were already PEGylated ex situ, a separate functionalization step on the sensor was not needed. The clearly larger signal response for the DOX liposome layer compared to the DOX+PEG liposome layer can be explained by the different penetration properties of the liposomes into the carboxymethyl dextran hydrogel matrix and interactions with the dodecyl lipid anchors depending on liposome properties. Lundquist and her colleagues have demonstrated that the hydrophilic PEG coating on liposomes decreases interactions with lipid anchors, preventing deep penetration into the carboxymethyl hydrogel matrix on the Biacore L1 chip [48]. The lower layer thickness for DOX+PEG liposomes could therefore be a result of smaller penetration PEGylated liposomes into the hydrogel, and stronger interaction of the plain DOX liposomes with the dodecyl moieties. It is important to note, however, that layer thicknesses and refractive indices cannot be interpreted solely by visual inspection of the sensorgrams. For example, the sensorgram in Figure 5 (B, II) shows a higher signal for DOX+PEG liposomes during soft corona formation, but in Table 2 (II) the average calculated soft corona layer thickness is slightly smaller for DOX+PEG liposomes. To explain these occasional abnormalities in signal behavior in brief, the modeled layer thickness $(d)$ and refractive index $(n)$ are dependent on the relative SPR peak minimum shift compared to the previous (lower) layer and its SPR peak minimum position, as well as the intensity fluctuations and SPR minimum peak width observed in the SPR angular spectra. Our modeling approach is a layer-by-layer evaluation procedure where the next layer is built by using the previous layer as a starting point for subsequent steps, in particular the protein corona determinations at II and III. Since the initial signal levels of DOX liposome immobilization compared to DOX+PEG are significantly higher (Figure 5, A), this difference can be expected to have a distorting effect on the subsequent protein corona modeling steps. Therefore, in addition to performing accurate modeling and calculations based on the full angular SPR curves and using data from several reproducible experiments, careful inspection of the signal levels at these different phases is needed in order to correctly interpret the phenomena taking place at the surface or in its near vicinity. Layer thicknesses and refractive indices for subsequent serum interactions were calculated as described previously. As previously perceived in the layer modeling process for OGD and OGD+PEG, hereby a similar observation can be made on the relatively good alignment between the measured and calculated SPR spectra. 


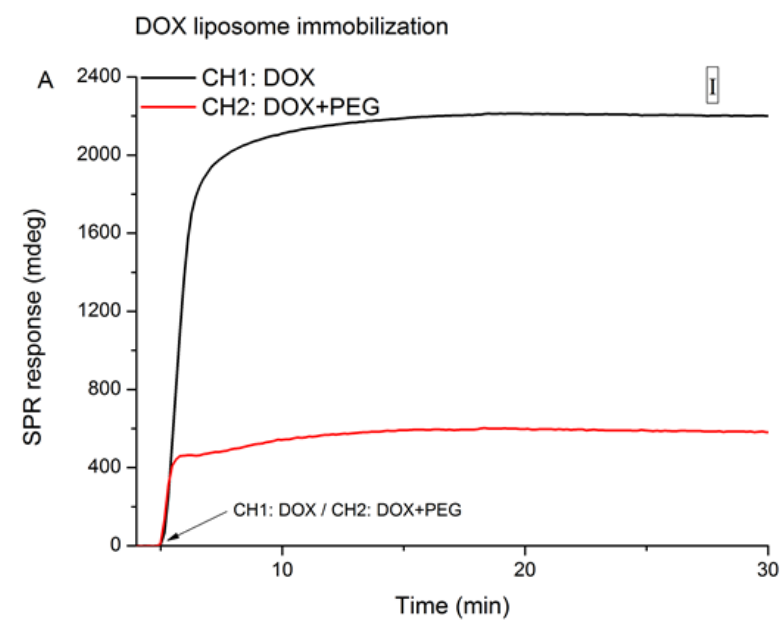

DOX liposome interaction with serum

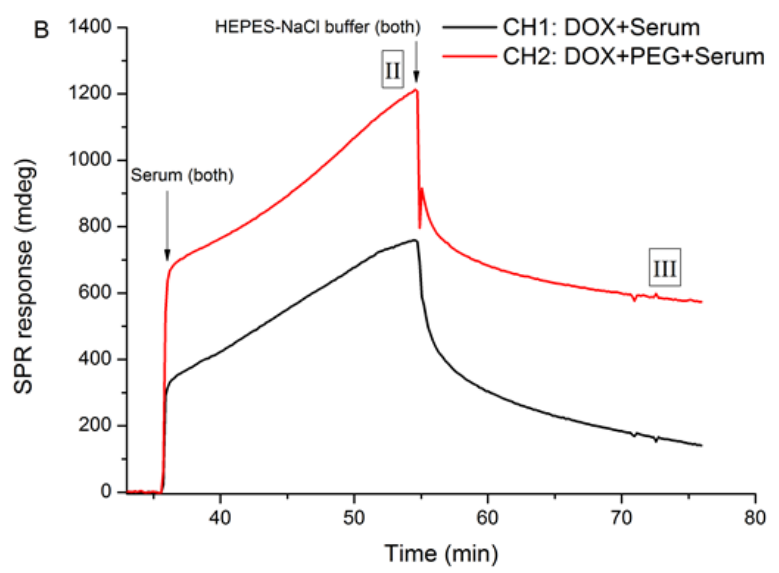

Fig. 5 MP-SPR sensorgram for DOX and DOX+PEG liposome immobilization and interaction with serum. A: Liposome immobilization onto the sensor surface with DOX in $\mathrm{CH} 1$ and DOX+PEG in $\mathrm{CH} 2$. The liposome layer thickness has been modeled and calculated at time point I. B: Serum interaction (from 35-55 min) with DOX (CH1) or DOX+PEG (CH2) liposomes at a flow speed of $100 \mu \mathrm{L} / \mathrm{min}$. Thickness $(d)$ and refractive index $(n)$ were modeled and calculated for soft corona (II) and hard corona (III) after restoration of buffer flow.
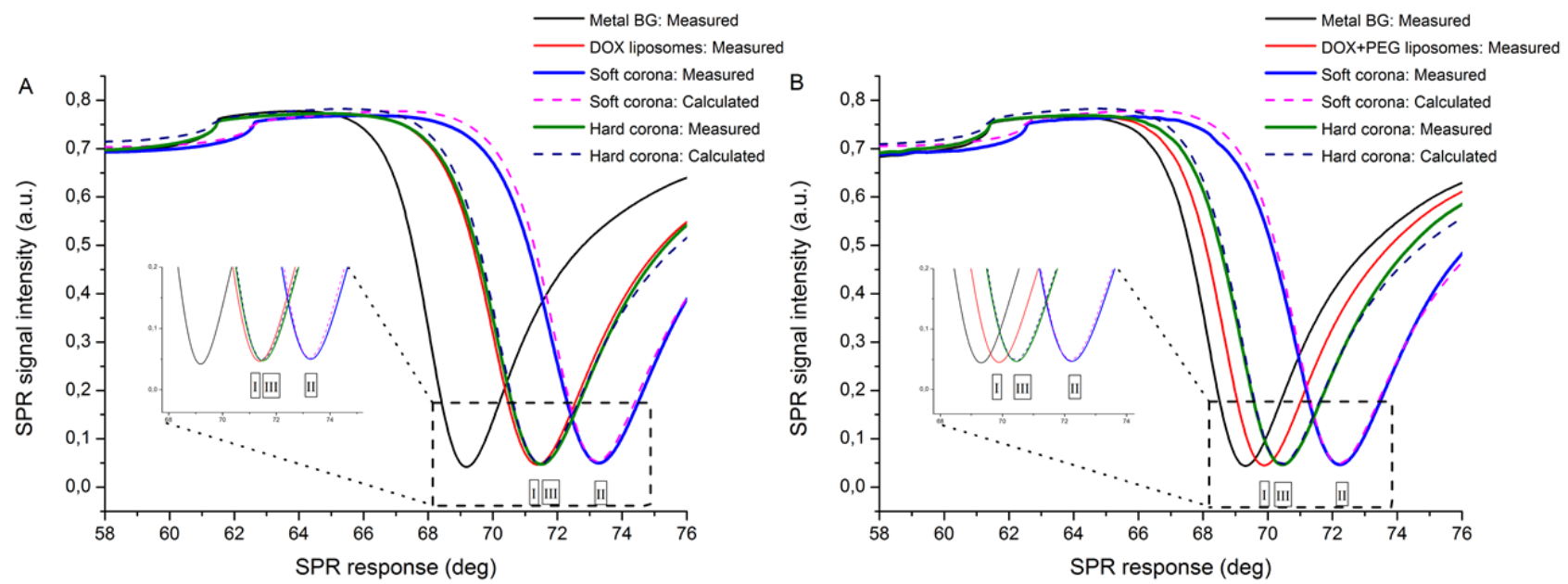

Fig. 6 Layer modeling for DOX and DOX+PEG liposomes using the measured full SPR angular spectra. A: SPR peak minimum shifts for the measured and modeled phases from the metal sensor surface (black line) to plain DOX (I) liposome immobilization, (II) soft corona, and (III) hard corona. B: Corresponding events for DOX+PEG.

Table 2. DOX and DOX+PEG liposomes and serum interaction results. The calculated values are low and high limits for layer thickness $(d)$ with corresponding refractive index $(n)$ values as averages ( \pm standard deviation) of three independent measurements for: (I) the immobilized DOX and DOX+PEG liposome layer; (II) soft protein corona formation following serum interaction; and (III) the residual hard corona after buffer flow restoration when a stable baseline is observed.

\begin{tabular}{|c|c|c|c|c|c|c|c|}
\hline & & \multicolumn{2}{|c|}{ (I) Liposome } & \multicolumn{2}{|c|}{ (II) Soft corona } & \multicolumn{2}{|c|}{ (III) Hard corona } \\
\hline \multirow{3}{*}{ 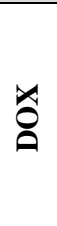 } & & Low & High & Low & High & Low & High \\
\hline & $d$ & $99.1 \pm 5.0$ & $153.2 \pm 8.2$ & $37.5 \pm 1.7$ & $86.8 \pm 19.6$ & $3.4 \pm 1.2$ & $6.6 \pm 1.9$ \\
\hline & $n$ & $\begin{array}{c}1.36237 \\
\pm 0.0010\end{array}$ & $\begin{array}{r}1.35680 \\
\pm 0.0009\end{array}$ & $\begin{array}{c}1.41742 \\
\pm 0.0046\end{array}$ & $\begin{array}{r}1.42930 \\
\pm 0.0175\end{array}$ & $\begin{array}{r}1.39440 \\
\pm 0.0034\end{array}$ & $\begin{array}{c}1.38614 \\
\pm 0.0063\end{array}$ \\
\hline \multirow{3}{*}{ 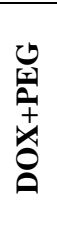 } & & Low & High & Low & High & Low & High \\
\hline & $d$ & $37.3 \pm 4.7$ & $49.1 \pm 2.9$ & $25.8 \pm 4.8$ & $60.0 \pm 18.0$ & $7.6 \pm 0.9$ & $13.0 \pm 0.8$ \\
\hline & $n$ & $\begin{array}{c}1.34971 \\
\pm 0.0017\end{array}$ & $\begin{array}{r}1.34722 \\
\pm 0.0002\end{array}$ & $\begin{array}{c}1.39711 \\
\pm 0.0069\end{array}$ & $\begin{array}{r}1.37730 \\
\pm 0.0026\end{array}$ & $\begin{array}{r}1.42353 \\
\pm 0.0075\end{array}$ & $\begin{array}{c}1.39187 \\
\pm 0.0085\end{array}$ \\
\hline
\end{tabular}

Refractive indices extracted during fitting procedure: serum 1.34960 , buffer 1.33535 . 
Table 2 represents the modeled and calculated values of DOX liposomes forming on average a 99.1-153.2 nm layer on the sensor surface with an average refractive index of 1.35680-1.36237. The immobilized DOX+PEG liposomes form a significantly thinner layer with an average of 37.3-49.1 and with a lower refractive index range of 1.34722-1.34971. We speculate that the exceedingly high signal produced by plain DOX liposomes is a consequence of its lipophilic interactions with the lipid anchors, promoted by the smaller size and absence of oligo-guanidyl moieties comparing to plain OGD liposomes, which are likely to lead to the formation of liposome multilayers instead of monolayers on the sensor surface $[45,48]$. After serum injection, a soft corona of 37.5-86.8 nm forms on DOX liposomes with a refractive index of 1.417421.42930, whereas the layer thickness on DOX+PEG is slightly thinner 25.8-60.0 $\pm 18.0 \mathrm{~nm}$. As with the liposome layer, the refractive index range of the DOX+PEG soft corona layer is lower 1.37730-1.39711. After restoration of buffer flow, a significantly thinner hard corona remains on DOX compared to DOX+PEG (3.4-6.6 vs. 7.6-13.0 nm). Interestingly, the refractive index of DOX hard corona is lower than the soft corona for the same liposome, or the hard corona observed on DOX+PEG (1.38614-1.39440 vs. 1.39187-1.42353, respectively). We hypothesize that these could be explained by a higher amount of light refracting species remaining at the vicinity of the surface, but may also be caused by events such as restructuring and phase transformations taking place in the biomolecular layers [49]. Based on the observations on the possible distorting effect of the significantly higher signal response in the DOX liposome immobilization step, contrasting DOX liposome results reliably with the three other liposome types would require further optimization of sensor saturation levels. Therefore, for proof-of-concept, we limit our discussion to compare protein corona formation on OGD and OGD+PEG to DOX+PEG as a "benchmark" liposome formulation.

Comparison of OGD and OGD+PEG with DOX+PEG serum interaction events. When the serum interaction events described in Tables 1 and 2 are compared, some important observations can be made. Firstly, refractive indices of soft corona layers on OGD and OGD+PEG liposomes are clearly smaller than the refractive indices of soft corona layers on DOX+PEG liposomes. This disparity is not dependent on the liposomes having PEG or not. This may be in relation to the differences in the properties of these liposome species, for example surface lipophilicity or hydrophilicity, surface charge and rheological properties, which translate into different compositions for the protein corona $[17,26]$. Secondly, OGD liposomes favor a soft corona layer formation that is slightly thicker and denser than in the case of OGD+PEG or DOX+PEG liposomes (34.2-106.0 vs. 22.2-63.2 and 25.8-60.0 nm, respectively). Absence of PEG increases the lipophilicity of the surface, and increased protein binding is expected [49]. Dynamic conditions may also contribute to this effect, as protein coronas formed on PEG-modified liposomes under dynamic conditions tends to be thicker than those resulting from static incubations, i.e. around $\sim 20-25 \mathrm{~nm}$ thicker [12]. For OGD and in a lesser extent for OGD+PEG, the presence of OGD oligo-guanidyl moieties and traces of endotoxin on the surface might also attract specific serum components, as we discussed earlier [15]. Thirdly and most interestingly, the plain OGD liposomes induce the formation of a thicker (9.6-16.4 nm) and denser (1.41121-1.44627) hard corona layer as a result of interaction with serum components compared to the other liposome samples. This is in line with the results of Walczyk et al.[13], who presented a range of 11-12 $\mathrm{nm}$ for "protein shell thickness" prior to extensive washing and around $10.5 \mathrm{~nm}$ after washing using differential centrifugal sedimentation of polystyrene model nanoparticles incubated for up to 6 hours with diluted plasma.

C3b interaction with immobilized OGD and OGD+PEG liposomes. Using MP-SPR, we determined the affinity and surface density mass of the opsonin C3b on the OGD and OGD+PEG liposome surfaces. This was done in order to gain insight whether the observed differences in the serum interaction studies could be explained at least in part by immunogenic properties of the nanocarriers - the increased tendency to recruit opsonins from the serum and, possibly, activate the complement system. C3b is a $180 \mathrm{kDa}$ fragment that results from the cleavage of anaphylatoxin C3a from complement component C3 [21,24]. C3b is able to covalently bind to amino or hydroxyl groups on artificial surfaces through its exposed thioester [21,24]. After immobilization of OGD or OGD+PEG liposomes to sensor surfaces in parallel channels, a series of $\mathrm{C} 3 \mathrm{~b}$ concentrations was injected into both channels: $0.00833,0.0833$, and 0.833 mol. As shown in Figure 7, a clearly larger stepwise SPR response signal is achieved in terms of C3b interacting with OGD surface without PEG (A) in comparison to OGD liposome surface with PEG $(\mathbf{B})$. The calculated results for deposited C3b surface mass densities were $\Gamma$ (total) $\sim 410-680 \mathrm{ng} / \mathrm{cm}_{2}$ for OGD liposomes without PEG, and $\Gamma$ (total) $\sim 120-200 \mathrm{ng} / \mathrm{cm} 2$ for OGD liposomes with PEG. These results are consistent with the findings of the serum interaction measurements. The calculated equilibrium dissociation constants for $\mathrm{C} 3 \mathrm{~b}$ match up reasonably well between the different liposome interaction schemes: for C3b interacting with OGD and OGD+PEG, $\mathrm{K}_{D, \mathrm{OGD}}=3.8 \times 10_{-8} \mathrm{M}$ and $\mathrm{K}_{D, \mathrm{OGD}+\mathrm{PEG}}=7.2 \times 10_{-8} \mathrm{M}$ were obtained, 
respectively. To the best of our knowledge, this is the first time the $\mathrm{C} 3 \mathrm{~b}$ affinities have been determined on biomaterial surfaces and on intact surface-immobilized liposomes.
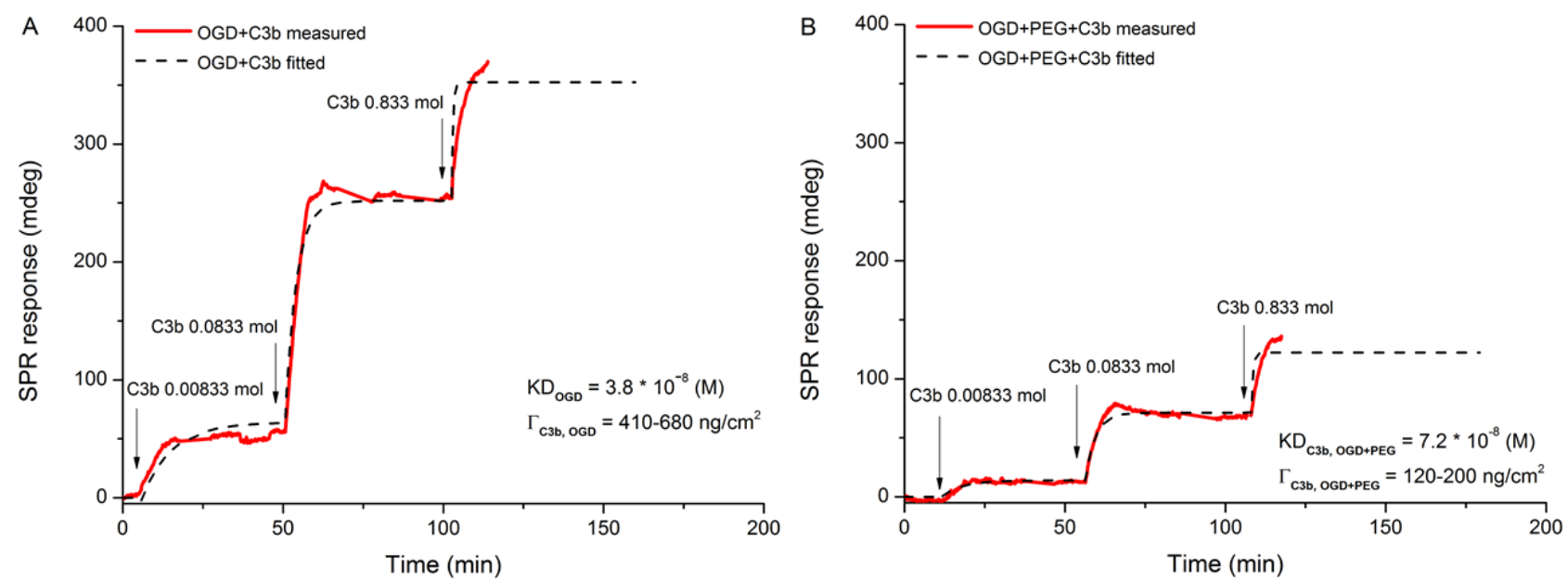

Fig. 7 C3b binding affinity and surface mass density onto OGD and OGD+PEG liposomes.

Based on these results, we demonstrate a connection between the tendency of the surface to adsorb serum components and a preference of $\mathrm{C} 3 \mathrm{~b}$ for the surface. Hence, the observed differences in interaction experiments of OGD liposomes with undiluted serum, as discussed above, may be in relation to complement system activation. In particular, the observed significant difference in the hard corona composition formed onto the OGD liposome layer without PEG may report that the OGD liposomes without PEG are capable of inducing more drastic opsonisation-related responses than the OGD liposomes with PEG. Unfortunately, since the OGD liposomes (but not the PEG added to functionalize these liposomes in situ to form OGD+PEG) used were shown to contain traces of endotoxins, it is not possible to reliably link increased opsonisation to PEG conformation or liposome properties at this stage. However, these observations merit further studies using other complement trigger molecules, as well as assays to confirm complement activation taking place at the surface, such as using antibodies specific to $\mathrm{C} 3 \mathrm{~b}$ degradation products such as $\mathrm{C} 3 \mathrm{c}$ [25].

In summary, we have demonstrated a convenient way to bind clinically relevant samples of nanocarriers to sensor surfaces and to study their interactions with full serum. Our focus was on intact liposomes that we modified with hydrophilic coating molecules before and after immobilization to sensor, which has not been reported previously. This method can be applied with relative ease to assess the effect of different surface modifications on unspecific protein binding with different types of nanocarriers. The main limitation in the present work was the use of serum instead of plasma; for instance, fibrinogen that is not present in serum is known to bind to PEG-coated liposomes [12]. This is explained in part by our interest in the complement activating properties of surface-immobilized liposomes, which cannot be studied in EDTA-anticoagulated plasma. We demonstrated that undiluted serum can be successfully used in the interaction studies, but we suggest that any blood product without cells - such as plasma or serum that has been depleted of some component or associated to a certain disease state - is equally suited for this experimental setup. In regard to the safety of nanocarriers, the possibility to link the production of secondary inflammatory mediators to surface events would provide complementary evidence of the immunogenic properties of nanocarriers. Even though it is difficult to replicate the in vivo conditions in blood circulation in vitro, the dynamic flow and temperature-controlled conditions improve the in vivo relevance of the presented method. Using MP-SPR and layer-by-layer modeling, we were able to separately identify distinct soft and hard coronas. The possibility to identify and elute these biomolecular layers from the surface of nanocarriers might provide interesting opportunities: for example, coupling MP-SPR technology to other analysis instruments such as LC-MS could permit dissecting subclasses of the protein corona, and producing snapshots of the protein corona at different times $[16,17,20]$.

\section{Conclusions}

In this study, we have developed a flexible, highly modifiable and sensitive one-instrument MP-SPR technology platform that can be used for interaction studies using undiluted serum with nanocarriers in controlled dynamic settings. We 
explored its essential capabilities with four different liposomes and conclude that MP-SPR is suitable for optical characterization of the formation and evolution of protein coronas on nanocarrier surfaces. Our reproducible measurements clearly demonstrated the formation of a distinct soft corona. In addition, we were able to determine binding affinity $\left(\mathrm{K}_{D}\right)$ for an essential opsonin, $\mathrm{C} 3 \mathrm{~b}$, directly on a biomaterial surface. Therefore, the described label-free platform provides a powerful preclinical tool for tuning nanocarrier surface architecture and composition to control protein corona formation.

\section{Compliance with ethical standards}

All the reported experiments are in compliance with current Finnish law. The authors declare no conflicts of interest. T.R. is affiliated with BioNavis Ltd., Ylöjärvi, Finland.

\section{References}

1. Shi J, Votruba AR, Farokhzad OC, Langer R. Nanotechnology in Drug Delivery and Tissue Engineering: From Discovery to Applications. Nano Lett. 2010;10:3223-30.

2. Nyström AM, Fadeel B. Safety assessment of nanomaterials: Implications for nanomedicine. J. Control. Release. Elsevier B.V.; 2012;161:403-8.

3. Bertrand N, Leroux J-C. The journey of a drug-carrier in the body: An anatomo-physiological perspective. J. Control. Release. Elsevier B.V.; 2012;161:152-63.

4. Knop K, Hoogenboom R, Fischer D, Schubert US. Poly(ethylene glycol) in drug delivery: Pros and cons as well as potential alternatives. Angew. Chemie - Int. Ed. 2010;49:6288-308.

5. Hamad I, Al-hanbali ḰO, Hunter AC, Rutt KJ, Andresen TL, Moghimi SM. Switching of Complement Activation Pathways at the Nanosphere Serum Interface : Implications for Stealth Nanoparticle Engineering. ACS Nano. 2010;4:6629-38.

6. Arima Y, Toda M, Iwata H. Complement activation on surfaces modified with ethylene glycol units. Biomaterials. 2008;29:551-60.

7. Salmaso S, Caliceti P. Stealth Properties to Improve Therapeutic Efficacy of Drug Nanocarriers. J. Drug Deliv. 2013;2013:1-19.

8. Mahon E, Salvati A, Baldelli Bombelli F, Lynch I, Dawson KA. Designing the nanoparticle-biomolecule interface for "targeting and therapeutic delivery." J. Control. Release. Elsevier B.V.; 2012;161:164-74.

9. Szebeni J, Muggia F, Gabizon A, Barenholz Y. Activation of complement by therapeutic liposomes and other lipid excipient-based therapeutic products: Prediction and prevention. Adv. Drug Deliv. Rev. Elsevier B.V.; 2011;63:102030.

10. Chanan-Khan A. Complement activation following first exposure to pegylated liposomal doxorubicin (Doxil(R)): possible role in hypersensitivity reactions. Ann. Oncol. 2003;14:1430-7.

11. Lynch I, Cedervall T, Lundqvist M, Cabaleiro-Lago C, Linse S, Dawson KA. The nanoparticle-protein complex as a biological entity; a complex fluids and surface science challenge for the 21 st century. Adv. Colloid Interface Sci. 2007;134-135:167-74.

12. Palchetti S, Colapicchioni V, Digiacomo L, Caracciolo G, Pozzi D, Capriotti AL, et al. The protein corona of circulating PEGylated liposomes. Biochim. Biophys. Acta - Biomembr. Elsevier B.V.; 2016;1858:189-96.

13. Walczyk D, Bombelli FB, Monopoli MP, Lynch I, Dawson K a. What the cell "sees" in bionanoscience. J. Am. Chem. Soc. 2010;132:5761-8.

14. Cedervall T, Lynch I, Lindman S, Berggård T, Thulin E, Nilsson H, et al. Understanding the nanoparticle-protein corona using methods to quantify exchange rates and affinities of proteins for nanoparticles. Proc. Natl. Acad. Sci. U. S. A. $2007 ; 104: 2050-5$.

15. Corbo C, Molinaro R, Parodi A, Furman NET, Salvatore F, Tasciotti E. The impact of nanoparticle protein corona on cytotoxicity, immunotoxicity and target drug delivery. Nanomedicine. 2016;11:81-100.

16. Docter D, Westmeier D, Markiewicz M, Stolte S, Knauer SK, Stauber RH. The nanoparticle biomolecule corona: lessons learned - challenge accepted? Chem. Soc. Rev. Royal Society of Chemistry; 2015;44:6094-121.

17. Tenzer S, Docter D, Kuharev J, Musyanovych A, Fetz V, Hecht R, et al. Rapid formation of plasma protein corona critically affects nanoparticle pathophysiology. Nat. Nanotechnol. 2013;8:772-81.

18. Lundqvist M, Stigler J, Elia G, Lynch I, Cedervall T, Dawson K a. Nanoparticle size and surface properties determine the protein corona with possible implications for biological impacts. Proc. Natl. Acad. Sci. U. S. A. 2008;105:14265-70.

19. Lundqvist M. Nanoparticles: Tracking protein corona over time. Nat. Publ. Gr. 2013;8:1-2.

20. Monopoli MP, Åberg C, Salvati A, Dawson KA, Åberg C, Salvati A, et al. Biomolecular coronas provide the biological identity of nanosized materials. Nat. Nanotechnol. Nature Publishing Group; 2012;7:779-86. 
21. Carroll M V, Sim RB. Complement in health and disease. Adv. Drug Deliv. Rev. Elsevier B.V.; $2011 ; 1-11$.

22. Pangburn MK, Schreiber RD, Müller-Eberhard HJ. C3b deposition during activation of the alternative complement pathway and the effect of deposition on the activating surface. J. Immunol. 1983;131:1930-5.

23. Andersson J, Ekdahl KN, Lambris JD, Nilsson B. Binding of C3 fragments on top of adsorbed plasma proteins during complement activation on a model biomaterial surface. Biomaterials. 2005;26:1477-85.

24. Nilsson B, Ekdahl KN, Mollnes TE, Lambris JD. The role of complement in biomaterial-induced inflammation. Mol. Immunol. 2007;44:82-94.

25. Arima Y, Toda M, Iwata H. Surface plasmon resonance in monitoring of complement activation on biomaterials. Adv. Drug Deliv. Rev. Elsevier B.V.; 2011;63:988-99.

26. Granqvist N, Yliperttula M, Välimäki S, Pulkkinen P, Tenhu H, Viitala T. Control of the morphology of lipid layers by substrate surface chemistry. Langmuir. 2014;30:2799-809.

27. Kretschmann E. Determination of optical constants of metals by excitation of surface plasmons. Zeitschrift Fur Phys. 1971;241:313-24.

28. Granqvist N, Liang H, Laurila T, Sadowski J, Yliperttula M, Viitala T. Characterizing ultrathin and thick organic layers by surface plasmon resonance three-wavelength and waveguide mode analysis. Langmuir. 2013;29:8561-71. 29. Bakhtiar R. Surface Plasmon Resonance Spectroscopy: A Versatile Technique in a Biochemist' s Toolbox. 2013; 30. Albers WM, Vikholm-lundin I. Surface Plasmon Resonance on Nanoscale Organic Films. 1988;83-125.

31. Liang H, Miranto H, Granqvist N, Sadowski JW, Viitala T, Wang B, et al. Surface plasmon resonance instrument as a refractometer for liquids and ultrathin films. Sensors Actuators, B Chem. Elsevier B.V.; 2010;149:212-20.

32. Abraham SA, Waterhouse DN, Mayer LD, Cullis PR, Madden TD, Bally MB. The liposomal formulation of doxorubicin. Methods Enzymol. 2005;391:71-97.

33. Bersani S, Salmaso S, Mastrotto F, Ravazzolo E, Semenzato A, Caliceti P. Star-like oligo-arginyl-maltotriosyl derivatives as novel cell-penetrating enhancers for the intracellular delivery of colloidal therapeutic systems. Bioconjug. Chem. 2012;23:1415-25.

34. Löfås S, Johnsson B. A novel hydrogel matrix on gold surfaces in surface plasmon resonance sensors for fast and efficient covalent immobilization of ligands. J. Chem. Soc. Chem. Commun. 1990;1526.

35. Peterlinz KA, Georgiadis R. Two-color approach for determination of thickness and dielectric constant of thin films using surface plasmon resonance spectroscopy. Opt. Commun. North-Holland; 1996;130:260-6.

36. and JHG, Georgiadis* RM. Temperature-Dependent Refractive Index Determination from Critical Angle

Measurements: Implications for Quantitative SPR Sensing. American Chemical Society ; 1999;

37. Zhou M, Otomo A, Yokoyama S, Mashiko S. Estimation of organic molecular film structures using surfaceplasmon resonance spectroscopy. Thin Solid Films. 2001;393:114-8.

38. Viitala T, Granqvist N, Hallila S, Raviña M, Yliperttula M. Elucidating the signal responses of multi-parametric surface plasmon resonance living cell sensing: a comparison between optical modeling and drug-MDCKII cell interaction measurements. PLoS One. Public Library of Science; 2013;8:e72192.

39. Granqvist N, Hanning A, Eng L, Tuppurainen J, Viitala T. Label-enhanced surface plasmon resonance: a new concept for improved performance in optical biosensor analysis. Sensors (Basel). Multidisciplinary Digital Publishing Institute (MDPI); 2013;13:15348-63.

40. Karlsson R, Fält A. Experimental design for kinetic analysis of protein-protein interactions with surface plasmon resonance biosensors. J. Immunol. Methods. 1997;200:121-33.

41. Zhao H, Brown PH, Schuck P. On the distribution of protein refractive index increments. Biophys. J. Biophysical Society; 2011;100:2309-17.

42. Ball V, Ramsden JJ. Buffer dependence of refractive index increments of protein solutions. Biopolymers. 1998;46:489-92.

43. Sadowski JW, Korhonen IKJ, Peltonen JPK. Characterization of thin films and their structures in surface plasmon resonance measurements. Opt. Eng. 1995;34:2581-6.

44. Kretschmann E, Raether H. Radiative decay of non-radiative surface plasmons excited by light. Z. Naturforsch. 1968;23:2135-6.

45. Hall D. Kinetic Models Describing Biomolecular Interactions at Surfaces. Handb. Surf. Plasmon Reson. 2008;81122.

46. Hamad I, Hunter a C, Szebeni J, Moghimi SM. Poly(ethylene glycol)s generate complement activation products in human serum through increased alternative pathway turnover and a MASP-2-dependent process. Mol. Immunol. 2008;46:225-32.

47. Gref R, Lück M, Quellec P, Marchand M, Dellacherie E, Harnisch S, et al. "Stealth" corona-core nanoparticles surface modified by polyethylene glycol (PEG): influences of the corona (PEG chain length and surface density) and of the core composition on phagocytic uptake and plasma protein adsorption. Colloids Surfaces B Biointerfaces.

2000;18:301-13.

48. Lundquist A, Hansen SB, Nordström H, Danielson UH, Edwards K. Biotinylated lipid bilayer disks as model membranes for biosensor analyses. Anal. Biochem. Elsevier Inc.; 2010;405:153-9.

49. Nel AE, Mädler L, Velegol D, Xia T, Hoek EM V, Somasundaran P, et al. Understanding biophysicochemical 
interactions at the nano-bio interface. Nat. Mater. Nature Publishing Group; 2009;8:543-57.

\section{Supplementary Materials}

Table S1. Physicochemical characterization and endotoxin determination results for DOX, DOX+PEG, OGD and OGD+PEG liposomes. Results are presented as average ( \pm standard deviation) for hydrodynamic diameter and $\zeta$-potential. Results for endotoxins are reported in EU/ml and described as positive (+) and negative (-) based on two complementary assays.

\begin{tabular}{|c|c|c|c|c|}
\hline & $\begin{array}{c}\text { Hydrodynamic } \\
\text { diameter }\end{array}$ & Polydispersity index & $\zeta$-potential & Endotoxin \\
\hline DOX & $98.12 \pm 14.80 \mathrm{~nm}$ & 0.324 & $-1.42 \pm 0.16 \mathrm{mV}$ & $<0.1 \mathrm{EU} / \mathrm{ml}(-)$ \\
\hline $\begin{array}{c}\text { DOX } \\
\text { +PEG }\end{array}$ & $105.07 \pm 2.64 \mathrm{~nm}$ & 0.017 & $-1.90 \pm 0.17 \mathrm{mV}$ & $<0.1 \mathrm{EU} / \mathrm{ml}(-)$ \\
\hline OGD & $227.03 \pm 19.28 \mathrm{~nm}$ & 0.254 & $+20.10 \pm 0.40 \mathrm{mV}$ & $>1.0 \mathrm{EU} / \mathrm{ml}(+)$ \\
\hline $\begin{array}{c}\text { OGD } \\
\text { +PEG }\end{array}$ & $248.43 \pm 30.40 \mathrm{~nm}$ & 0.328 & $-1.84 \pm 0.41 \mathrm{mV}$ & $>1.0 \mathrm{EU} / \mathrm{ml}(+) \mathrm{a}$ \\
\hline
\end{tabular}

a) OGD+PEG liposome tested after the charge-to-charge association process tested positive for endotoxin, but the polyanionic-PEG block-copolymer solution alone contained $<0.1 \mathrm{EU} / \mathrm{ml}$ of endotoxin.

Table S2. The compiled modeling results of separate experiments for OGD and OGD+PEG liposomes and serum interactions. The calculated values are low and high limits for layer thickness $(d)$ with corresponding refractive index $(n)$ values from all three independent measurements and their average $( \pm$ standard deviation). Refractive indices extracted during fitting procedure: serum 1.34960 , buffer 1.33535 .

\begin{tabular}{|c|c|c|c|c|c|c|c|}
\hline & \multicolumn{3}{|c|}{ (I) Liposome } & \multicolumn{2}{|c|}{ (II) Soft corona } & \multicolumn{2}{|c|}{ (III) Hard corona } \\
\hline \multirow{9}{*}{0} & & Low & High & Low & High & Low & High \\
\hline & 1 & 39.97 & 51.08 & 32.7 & 94.28 & 9.73 & 16.0 \\
\hline & 2 & 31.31 & 42.15 & 33.37 & 97.72 & 10.5 & 17.45 \\
\hline & 3 & 49.88 & 73.27 & 36.61 & 126.05 & 8.59 & 15.79 \\
\hline & $d$ & $\begin{array}{c}40.3867 \pm \\
9.29201 \\
\end{array}$ & $55.5 \pm 16.0239$ & $\begin{array}{c}34.2267 \pm \\
2.09104 \\
\end{array}$ & $\begin{array}{c}106.017 \pm \\
17.4344 \\
\end{array}$ & $\begin{array}{c}9.60667 \pm \\
0.96095 \\
\end{array}$ & $16.4133 \pm 0.9039$ \\
\hline & 1 & 39.97 & 1.35166 & 1.40034 & 1.37681 & 1.44632 & 1.41075 \\
\hline & 2 & 31.31 & 1.34876 & 1.40279 & 1.37927 & 1.45346 & 1.41491 \\
\hline & 3 & 49.88 & 1.34947 & 1.38789 & 1.37619 & 1.43904 & 1.40797 \\
\hline & $n$ & $\begin{array}{l}1.35302 \\
\pm 0.0021\end{array}$ & $\begin{array}{c}1.34996 \\
\pm 0.00151\end{array}$ & $\begin{array}{c}1.39701 \\
\pm 0.00799\end{array}$ & $\begin{array}{c}1.37742 \\
\pm 0.00163\end{array}$ & $\begin{array}{c}1.44627 \\
\pm 0.0072\end{array}$ & $\begin{array}{r}1.41121 \\
\pm 0.0035\end{array}$ \\
\hline \multirow{4}{*}{ 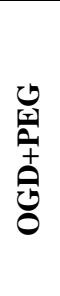 } & & Low & High & Low & High & Low & High \\
\hline & 1 & 48.0 & 61.94 & 22.63 & 54.75 & 3.2 & 4.99 \\
\hline & 2 & 45.44 & 68.51 & 36.53 & 124.19 & 7.73 & 15.99 \\
\hline & 3 & 39.72 & 52.35 & 7.5 & 10.64 & 2.99 & 4.8 \\
\hline
\end{tabular}




\begin{tabular}{|c|c|c|c|c|c|c|}
\hline $\boldsymbol{d}$ & $\begin{array}{c}44.3867 \\
\pm 4.23931\end{array}$ & $\begin{array}{c}60.9333 \\
\pm 8.1269\end{array}$ & $22.22 \pm 14.5193$ & $\begin{array}{c}63.1933 \pm \\
57.2439\end{array}$ & $4.64 \pm 2.67808$ & $8.6 \pm 6.4$ \\
\hline 1 & 1.35643 & 1.35295 & 1.38403 & 1.37284 & 1.3802 & 1.36849 \\
\hline 2 & 1.35987 & 1.35461 & 1.39426 & 1.38047 & 1.43979 & 1.40062 \\
\hline 3 & 1.35406 & 1.35034 & 1.35963 & 1.38709 & 1.36573 & 1.35681 \\
\hline \hline $\boldsymbol{n}$ & $\begin{array}{c}1.35679 \\
\pm 0.00292\end{array}$ & $\begin{array}{c}1.35263 \\
\pm 0.00215\end{array}$ & $\begin{array}{c}1.37931 \\
\pm 0.01779\end{array}$ & $\begin{array}{c}1.38013 \\
\pm 0.00713\end{array}$ & $\begin{array}{c}1.39524 \\
\pm 0.03925\end{array}$ & $\begin{array}{c}1.37531 \\
\pm 0.02269\end{array}$ \\
\hline
\end{tabular}

Table S3. The compiled modeling results of separate experiments for DOX and DOX+PEG liposomes and serum interactions. The calculated values are low and high limits for layer thickness $(d)$ with corresponding refractive index $(n)$ values from all three independent measurements and their average ( \pm standard deviation). Refractive indices extracted during fitting procedure: serum 1.34960 , buffer 1.33535 .

\begin{tabular}{|c|c|c|c|c|c|c|c|}
\hline & \multicolumn{3}{|c|}{ (I) Liposome } & \multicolumn{2}{|c|}{ (II) Soft corona } & \multicolumn{2}{|c|}{ (III) Hard corona } \\
\hline \multirow{9}{*}{ ڤ̊ } & & Low & High & Low & High & Low & High \\
\hline & 1 & 104.01 & 161.5 & 39.48 & 88.5 & 3.85 & 7.45 \\
\hline & 2 & 93.98 & 145.02 & 36.32 & 105.39 & 1.98 & 4.39 \\
\hline & 3 & 99.16 & 153.05 & 36.63 & 66.41 & 4.26 & 7.81 \\
\hline & $d$ & $99.05 \pm 5.015905$ & $\begin{array}{l}153.19 \pm \\
8.240892\end{array}$ & $\begin{array}{c}37.47667 \pm \\
1.741848\end{array}$ & $\begin{array}{c}86.76667 \pm \\
19.54772\end{array}$ & $\begin{array}{c}3.363333 \pm \\
1.215415\end{array}$ & $6.55 \pm 1.879255$ \\
\hline & 1 & 1.36309 & 1.35754 & 1.42127 & 1.4351 & 1.39668 & 1.39222 \\
\hline & 2 & 1.36128 & 1.35579 & 1.42137 & 1.40966 & 1.39603 & 1.37973 \\
\hline & 3 & 1.36275 & 1.35706 & 1.41862 & 1.44315 & 1.39048 & 1.38648 \\
\hline & $n$ & $\begin{array}{c}1.362373 \\
\pm 0.000962\end{array}$ & $\begin{array}{l}1.356797 \\
\pm 0.0009\end{array}$ & $\begin{array}{c}1.41742 \\
\pm 0.00457\end{array}$ & $\begin{array}{c}1.429303 \\
\pm 0.017481\end{array}$ & $\begin{array}{c}1.39440 \\
\pm 0.00341\end{array}$ & $\begin{array}{c}1.38614 \\
\pm 0.00625\end{array}$ \\
\hline \multirow{9}{*}{ 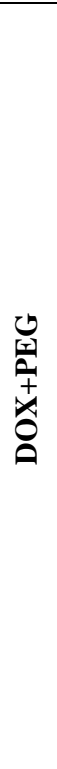 } & & Low & High & Low & High & Low & High \\
\hline & 1 & 40.8 & 50.64 & 20.27 & 51.26 & 6.65 & 12.17 \\
\hline & 2 & 31.98 & 45.78 & 27.62 & 48.07 & 8.25 & 13.57 \\
\hline & 3 & 39.2 & 50.87 & 29.36 & 80.68 & 7.96 & 13.36 \\
\hline & $d$ & $\begin{array}{c}37.3267 \pm \\
4.69895\end{array}$ & $\begin{array}{c}49.0967 \pm \\
2.87462\end{array}$ & $25.75 \pm 4.8249$ & $\begin{array}{c}60.0033 \pm \\
17.9774\end{array}$ & $7.62 \pm 0.85247$ & $\begin{array}{c}13.0333 \pm \\
0.75501\end{array}$ \\
\hline & 1 & 1.34788 & 1.34742 & 1.40478 & 1.38021 & 1.41521 & 1.38219 \\
\hline & 2 & 1.35118 & 1.34706 & 1.39126 & 1.37628 & 1.42977 & 1.39814 \\
\hline & 3 & 1.35008 & 1.34719 & 1.39528 & 1.3754 & 1.42562 & 1.39527 \\
\hline & $n$ & $\begin{array}{c}1.34971 \\
\pm 0.00168\end{array}$ & $\begin{array}{c}1.34722 \\
\pm 0.00018\end{array}$ & $\begin{array}{c}1.39711 \\
\pm 0.00694\end{array}$ & $\begin{array}{c}1.37730 \\
\pm 0.00256\end{array}$ & $\begin{array}{r}1.42353 \\
\pm 0.0075\end{array}$ & $\begin{array}{r}1.39187 \\
\pm 0.0085\end{array}$ \\
\hline
\end{tabular}

